and perfection, and the optimization of growth conditions.

With such unparalleled cleanliness, the $\mathrm{ZnO}$ heterostructures prepared by Tsukazaki and colleagues are not only in the running for exploring the fundamental physics of high mobility in more ionic electronic systems, but might soon become relevant to the same application that has been tantalizing architects of quantum computers and motivating FQHE growers to find ways of breaking the 100 million mobility barrier in GaAs-based 2DEGs. This involves the non-Abelian statistics predicted to accompany the unusual 5/2 FQHE state $^{11}$. If non-Abelian statistics do indeed accompany the $5 / 2$ state, it could be a route to a topological quantum computer with the same advantages for breaking codes as other quantum computing architectures being actively pursued, but much more amenable to lithography and scaling. This state, which so far has only been cleanly seen in the highest mobility GaAs 2DEGs, might be observable in $\mathrm{ZnO}$ 2DEGs with additional mobility improvements combined with cooling the electron gas to $10 \mathrm{mK}$ or lower.

Nevertheless, the sudden worsening of the magnetotransport in the $\mathrm{ZnO}$ as its 2DEG carrier density is lowered indicates that inhomogeneities in the sample are present, and these will need to be suppressed. Given the incredible rate of progress achieved by Tsukazaki and colleagues, $\mathrm{ZnO}$ could possibly go from its medicinal uses as calamine lotion to reduce itching, or that messy stuff you dreaded having rubbed on your nose as a kid to prevent sunburn, to being the pristine cornerstone of quantum computer technology.
Darrell G. Schlom is in the Department of Materials Science and Engineering, Cornell University, Ithaca, New York 14853-1501, USA. Loren N. Pfeiffer is in the Department of Electrical Engineering, Princeton University, Princeton, New Jersey 08544, USA.

e-mail:schlom@cornell.edu

\section{References}

1. Tsukazaki, A. et al. Nature Mater. 9, 889-893 (2010).

2. Tsui, D. C., Stormer, H. L. \& Gossard, A. C. Phys. Rev. Lett. 48, 1559-1562 (1982)

. De Poortere, E. P. et al. Appl. Phys. Lett. 80, 1583-1585 (2002).

4. Nelson, S. F. et al. Appl. Phys. Lett. 61, 64-66 (1992)

5. Manfra, M. J. et al. J. Appl. Phys. 92, 338-345 (2002).

6. Du, X., Skachko, I., Duerr, F., Luican, A. \& Andrei, E. Y. Nature 462, 192-195 (2009).

. Furneaux, J. E. et al. Surf. Sci. 170, 154-159 (1986).

8. Look, D. C. Solid State Commun. 105, 399-401 (1998).

9. Tsukazaki, A. et al. Science 315, 1388-1391 (2007)

10. Umansky, V. et al. J. Cryst. Growth 311, 1658-1661 (2009).

11. Das Sarma, S., Freedman, M. \& Nayak, C. Phys. Rev. Lett. 94, 166802 (2005).

12. Pfeiffer, L. \& West, K. W. Physica E 20, 57-64 (2003)

\title{
BEANBAG ROBOTICS
}

It's a commonplace observation in robotic engineering that some of the hardest tasks for robots are the ones we do without thinking: walking without falling over, say, or catching a ball. Even the simple feat of picking up objects, when considered as a problem in control systems engineering, becomes a formidable challenge. How should we position the fingers on approach, where should we grip the object, how much pressure should we apply? Answering these questions generally requires exquisite feedback between vision, motor control and tactile sensing, not to mention (in our case) a fair degree of intuition and training.

The ingenuity that has gone into solving these problems in robotics is exhilarating, as exemplified by the very recent reports in this journal of pressure-sensing 'smart skin', ${ }^{1,2}$. But these solutions tend to be predicated on the assumption that a robotic hand will follow the human prototype in having several gripping fingers. The widespread use of this design in the animal world testifies to its virtues, but there's no escaping the demands it makes on actuation, sensing and feedback.

Now Eric Brown of the University of Chicago and his co-workers have described a new design for a robotic gripper that dispenses altogether with these difficulties by replacing active control with passive adaptability. Their device has no fingers at all, but instead uses a soft mass that moulds itself to the shape of the object to be gripped ${ }^{3}$. The crucial aspect of the design is that, once configured in this way simply by pressing onto the object, the gripper undergoes a transition from soft to hard, becoming a rigid body encasing enough of the object to hold it with, in general, an appreciable force.

That is achieved by filling the body of the gripper - an elastic latex bag - with granular material, such as tiny glass spheres or, in one prototype, ground coffee. Rigidification of the conformable grainy mass is then induced by evacuating the air between the grains, causing slight compaction. This is sufficient to trigger a jamming transition: the grains enter a collective state of immobility, like that in a blocked funnel, which, as Brown's co-author Heinrich Jaeger explains in another preprint ${ }^{4}$, is a non-equilibrium state directly analogous to a glass. Indeed, such a packing-induced transition between solidity and fluidity is familiar to anyone who has ever opened a vacuum-packed packet of coffee.

Once rigid, the gripper holds an object by a combination of three

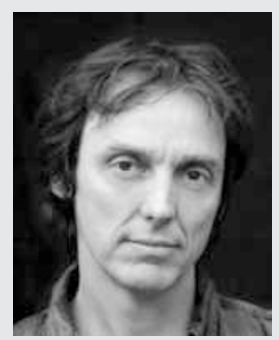

PHILIP BALL

mechanisms: friction, suction caused by deformation of the jammed bag as it lifts, and geometrical 'wrap-around' interlocking. The resultant gripping force depends on the geometry of the object, but a whole variety of forms, from steel springs to raw eggs, can be securely held. What is more, the device works in the wet, and can grip several different objects at once while retaining their orientation. Much as in the case of walking robots ${ }^{5}$, it shows how smart use of passive control can greatly simplify the engineering problem.

References

1. Takei, K. et al. Nature Mater. 9, 821-826 (2010).

2. Mannsfeld, S. C. B. et al. Nature Mater. 9, 859-864 (2010)

3. Brown, E. et al. preprint http://www.arxiv.org/ abs/1009.4444 (2010).

4. Jaeger, H. \& Liu, A. J. preprint http://www.arxiv.org/ abs/1009.4874 (2010).

5. Collins, S. H.,Wisse, M., Ruina, A. \& Tedrake, R. Science 307, 1082-1085 (2005). 